\title{
Instabilities in massive stars
}

\author{
Stanley P. Owocki \\ Bartol Research Institute, University of Delaware, Newark, DE 19716, \\ $U S A$
}

\begin{abstract}
A defining property of massive stars is the dominant, dynamical role played by radiation throughout the stellar interior, atmosphere, and wind. Associated with this radiation hydrodynamics are several distinct kinds of instabilities that can lead to convection in both core and envelope, clumping in atmosphere and wind outflow, and perhaps even the dramatic mass loss outbursts associated with Luminous Blue Variable phases. Here I review these instabilities with emphasis on basic physical properties of radiative driving. I draw on two specific examples of dynamical instability, namely the strong instability associated with line-driving of a stellar wind outflow, and the global stellar instabilities associated with approaching or exceeding a modified Eddington limit. I conclude with a brief mention of recent ideas on the role of stellar rotation in the shaping of bipolar LBV outbursts.
\end{abstract}

\section{Radiative force and the Eddington limit}

The cores of massive stars have long been recognized to be unstable to convection, and even to eventual collapse and supernova explosion once nuclear fuels have been exhausted. But in recent years there has been a growing appreciation that their envelopes, atmospheres, and winds may also be subject to a variety of instabilities, rooted generally in the strong dynamical role of radiative momentum in these characteristically very luminous stars. To focus the present review on these dynamical instabilities, let us begin by considering the general form for the radiative acceleration $g_{\mathrm{rad}}$ associated with a specific opacity $\kappa_{\nu}$ (also known as the mass absorption coefficient, with CGS units $\mathrm{cm}^{2} \mathrm{~g}^{-1}$ ) for interaction of stellar material with a radiative flux $F_{\nu}$ at photon frequency $\nu$,

$$
g_{\mathrm{rad}}=\int_{0}^{\infty} d \nu \kappa_{\nu} F_{\nu} / \mathrm{c}
$$

with $\mathrm{c}$ the speed of light. In general the opacity $\kappa_{\nu}$ includes both broad-band continuum processes - e.g., Thomson scattering of electrons, and bound-free or free-free absorption - and bound-bound transitions associated with line absorption and/or scattering.

In the static envelope and atmosphere, the reduction in flux in saturated lines keeps the associated line-force small, and so to a good approximation the overall radiative acceleration is dominated by a continuum contribution characterized by electron scattering. Because such opacity is nearly gray (frequencyindependent), it can be pulled outside the frequency integration in eqn.(1). 
In an idealized, spherically symmetric, radiative envelope, the bolometric flux $F \equiv \int_{0}^{\infty} d \nu F_{\nu}$ is thus purely radial, and at any local radius $r$ is simply set by $F=L / 4 \pi r^{2}$, where $L$ is the total bolometric luminosity generated in the stellar core. The radiative acceleration associated with such a gray opacity $\kappa$ is thus given by

$$
g_{\mathrm{rad}}=\kappa F / \mathrm{c}=\frac{\kappa L}{4 \pi r^{2} \mathrm{c}} \equiv \Gamma g,
$$

where the last equality introduces the Eddington parameter $\Gamma \equiv \kappa L / 4 \pi \mathrm{G} M \mathrm{c}$, which gives the ratio of the radiative acceleration to the local gravitational acceleration, $g \equiv \mathrm{G} M / r^{2}$, with $\mathrm{G}$ the gravitation constant and $M$ the stellar mass. A key point is that, since both gravity and radiative flux have the same $r^{-2}$ decline with radius $r$, this ratio can often be considered nearly independent of radius, that is when the opacity $\kappa$, radiative luminosity $L$, and mass $M$ are all fixed. (As discussed below, there are various circumstances in which this is not the case.) For the classical case of pure electron scattering, the Eddington parameter scales as

$$
\Gamma_{\mathrm{e}}=2.6 \times 10^{-5} \frac{L}{\mathrm{~L}_{\odot}} \frac{\mathrm{M}_{\odot}}{M}
$$

Because stellar luminosity generally scales with a high power of the stellar mass, i.e., $L \propto \mathrm{M}^{3-4}$, massive stars with $M>10 \mathrm{M}_{\odot}$ generally have electron Eddington parameters of order $\Gamma_{e} \simeq 0.1-1$. Indeed, $\Gamma_{e} \equiv 1$ defines an Eddington limit, for which the entire star would become unbound, at least in this idealized model of 1-D, spherically symmetric, radiative envelope. It should thus be emphasized that this does not represent an appropriate condition for the steady-state mass loss characteristic of a stellar wind, since that requires an outwardly increasing radiative force that goes from being less than gravity in a bound stellar envelope to exceeding gravity in the outflowing stellar wind.

\section{Line-driven stellar winds}

The resonant nature of line absorption leads to an opacity that is inherently much stronger than from free electrons. For example, in the somewhat idealized, optically thin limit that all the line opacity could be illuminated with a flat, unattenuated continuum spectrum with the stellar luminosity, the total lineforce would exceed the free-electron force by a huge factor, of order $Q \approx 1000$ (Gayley 1995). For massive stars with typical electron Eddington parameters just below unity, this implies a net outward line acceleration that could be as high as $\Gamma_{\text {lines }} \approx 1000$ times the acceleration of gravity!

Of course, this does not generally occur in practice, because of the selfabsorption of the lines. Within the standard Castor, Abbott, and Klein (1975; hereafter CAK) line-driven wind theory, the number distribution $N$ of spectral lines is approximated as a power law in opacity, $\kappa d N / d \kappa=\left(\kappa / \kappa_{\mathrm{o}}\right)^{\alpha-1}$, where the CAK power index $\alpha \simeq 0.5-0.7$, and the normalization $\kappa_{\mathrm{o}} \simeq Q \kappa_{\mathrm{e}}$. The lineensemble radiation force is then reduced by a factor $1 / \tau_{\mathrm{o}}^{\alpha}$, i.e., $\Gamma_{\text {lines }}=Q \Gamma_{\mathrm{e}} / \tau_{\mathrm{o}}^{\alpha}$, where $\tau_{\mathrm{o}}=Q \kappa_{\mathrm{e}} \rho \mathrm{c} /(d v / d r)$ is the Sobolev optical thickness for the normalization opacity $\kappa_{\mathrm{o}}$. The CAK mass loss is roughly that which makes the outward line force just sufficient to overcome the electron scattering reduced gravity, i.e., with 
$\Gamma_{\text {lines }} \simeq 1-\Gamma_{\mathrm{e}}$

$$
\dot{M}_{c a k} \approx \frac{L}{\mathrm{c}^{2}}\left[\frac{Q \Gamma_{\mathrm{e}}}{1-\Gamma_{\mathrm{e}}}\right]^{-1+1 / \alpha}
$$

where we have used the definition of the mass loss rate $\dot{M} \equiv 4 \pi \rho v r^{2}$ and the fact that for such a CAK solution, $v d v / d r \simeq g\left(1-\Gamma_{\mathrm{e}}\right)$. This last property further yields the characteristic CAK velocity law scaling $v(r) \simeq v_{\text {esc }}(1-R / r)^{1 / 2}$, where $v_{\text {esc }} \equiv\left(\mathrm{G} M\left(1-\Gamma_{e}\right) / R\right)^{1 / 2}$ is the effective escape speed.

\subsection{Line de-shadowing instability}

Even though the mean properties of a line-driven wind are thus characterized by an outward acceleration comparable to the inward acceleration of gravity, the latent potential for the optically thin line acceleration to be a huge factor $Q \simeq 10^{3}$ larger implies a capacity for an intrinsically strong 'line de-shadowing' instability. Quantitative linear instability analyses have been carried out by MacGregor et al. (1979), Carlberg (1980), and Owocki \& Rybicki (1984, 1985), but the qualitative identification of this instability dates back to discussions by Milne (1926) and Lucy \& Solomon (1970). An optically thin velocity perturbation $\delta v$ Doppler shifts the local line opacity out of the absorption shadow of preceding flow, leading to a perturbed line-force that scales with the perturbed velocity, $\delta g_{1} / g_{1} \simeq \delta v / v_{\text {th }}$, where $v_{\text {th }}$ is the ion thermal velocity that sets the width of the line-profile. This leads to very strong instability, with characteristic growth rate $\Omega \simeq g_{\mathrm{l}} / v_{\text {th }}$ which is of order $v / v_{\text {th }} \approx 100$ faster than a typical wind expansion rate, $d v / d r$. The instability applies to optically thin perturbations with length scale $\lambda$ near or below the Sobolev length $L_{\mathrm{Sob}}=v_{\text {th }} /(d v / d r)$, but vanishes in the Sobolev limit $\lambda \gg L_{\mathrm{Sob}}$, for which the perturbed force equals the velocity gradient $\delta g_{1} / g_{1} \simeq \delta v^{\prime} / v^{\prime}$ (Abbott 1980; Owocki \& Rybicki 1984). The growth rate is moreover somewhat reduced, especially near the stellar surface, by a 'line-drag' of the diffuse, scattered radiation (Lucy 1984; Owocki \& Rybicki 1985).

For numerical simulations of the nonlinear evolution of this instability, a key challenge is in developing computationally tractable treatments of the inherently non-local dependence of the non-Sobolev line-force, including both the direct absorption (Owocki, Castor \& Rybicki 1987) and the diffuse components (Owocki 1991; Owocki \& Puls 1996, 1999). An excellent overview is given in the habilitation thesis by Feldmeier (2001). For 1-D instability simulations done so far, the average flow still follows the usual CAK solution, but develops an extensive structure characterized by dense clumps that are compressed by strong reverse shocks from must faster, rarefied flow. The extensive clumping is of potentially great importance for interpretation of density-squared emission diagnostics, including both $\mathrm{H} \alpha$ emission near the star, and the infrared and radio emission in the distant wind (Runacres \& Owocki 2002). Models with a full energy treatment suggest that the observed X-ray flux from such massive stars might arise from forward shocks that form from collisions between the dense clumps (Feldmeier et al. 1997), and the porosity of the medium may also be important for X-ray line profiles and variability (Oskinova et al. 2001, 2003). Overall, it seems the instability might well be the source of the compressive turbulence that has been conjectured to explain migrating features in optical emission lines from Wolf- 
Rayet stars (Lépine et al. 1996; Lépine \& Moffat 1999). However, only a few tentative attempts have been made so far to model the multidimensional turbulent structure that is likely to arise from this strong line-deshadowing instability (Owocki 1999; Dessart \& Owocki 2002; Gómez \& Williams, these Proceedings).

\subsection{Large-scale wind structure from surface perturbation}

In addition to such small-scale, stochastic, turbulent structure, line-driven stellar winds show evidence of much larger scale structure, e.g., as manifest in episodic Discrete Absorption Components (DACs) and Periodic Absorption Modulations (PAMs) in UV wind lines. The latter seem most likely to arise from wind perturbation by radial or non-radial pulsation, while the former may arise from mass ejections that may be associated with surface magnetic fields. Many of the properties of recurring DACs and PAMs are well produced by dynamical models of Co-rotating Interaction Regions (CIRs) that arise from a generic modulation of radiative driving near the surface of a rotating star (Cranmer \& Owocki 1996). Initial dynamical simulations linked specifically to surface pulsation are given in Cranmer (1996) and Owocki \& Cranmer (2002). For magnetic channeling of line-driven outflows, some initial analyses were done by Babel \& Montmerle (1997), with recent extensions to full MHD simulation by ud-Doula \& Owocki (2002). The possible disruption of large-scale flow structure by the small-scale intrinsic instability was examined by Owocki (1999). Finally, we note that the interactions between flow streams are themselves subject to many kinds of instability. (See, e.g., summary by Pittard 2000).

\section{Continuum radiation force and instability}

Let us now return to dynamical issues associated with continuum opacity in the stellar envelope and atmosphere. Below the Eddington limit, the envelope stratification is given in terms of a hydrostatic equilibrium that can be cast in the form

$$
\frac{d P_{\text {tot }}}{d \tau}=\frac{F_{\text {crit }}}{\mathrm{c}}
$$

where $d \tau \equiv \kappa \rho d r$ defines an optical depth scale in terms of the mass density $\rho$, $P_{\text {tot }}=P_{\text {rad }}+P_{\text {gas }}$ is the total pressure from radiation and gas, and $F_{\text {crit }}=g \mathrm{c} / \kappa$ is the critical radiation flux for which $\Gamma=1$. The advantage to writing eqn. (5) in this form is that it is then quite similar to the equation for radiative diffusion, which sets the the stratification of radiation pressure in optically thick regions with $\tau \gg 1$,

$$
\frac{d P_{\mathrm{rad}}}{d \tau}=\frac{F_{\mathrm{rad}}}{\mathrm{c}},
$$

where $F_{\text {rad }}$ is the radiative flux. Away from the optically thin surface layers that set detailed boundary values, integration of eqns. (5) and (6) thus implies

$$
\frac{P_{\text {rad }}}{P_{\text {tot }}}=\Gamma ; \tau \gg 1 \text {. }
$$

For the optically thick stellar envelope and/or deep atmosphere, this shows that the relative importance of radiation to gas pressure scales as $P_{\mathrm{rad}} / P_{\mathrm{gas}}=$ $\Gamma /(1-\Gamma)$, which thus diverges toward the Eddington limit $\Gamma \rightarrow 1$. 


\subsection{Convective instability of the Eddington limit}

Actually, using arguments based on equations (4) and (5), Joss et al. (1973) showed that an envelope approaching the Eddington limit would necessarily first become convectively unstable, even in the absence of the kind of strong opacity bump associated with convective recombination layers in lower-mass stars. This finding has quite profound and widespread implications for the interior and envelope structure of massive stars. For example, even such a canonical O-type supergiant like $\zeta$ Pup is estimated to have an Eddington parameter $\Gamma_{e} \simeq 0.5$. Thus, since the luminosity is generated at very small radii within the core, while the mass distribution $M(r)$ is more extended, then even with a fixed opacity $\kappa$, the local Eddington parameter increases inward as $\Gamma(r)=\Gamma_{*} M\left(R_{*}\right) / M(r)$, where $\Gamma_{*}$ is the Eddington parameter at surface $r=R_{*}$. In the case of $\zeta$ Pup with $\Gamma_{*} \simeq 0.5$, we thus expect that the $50 \%$ of its innermost mass should become convectively unstable because of locally exceeding the Eddington limit!

For the high-density of such a deep interior, the efficiency of convection means that the convective energy flux $F_{\text {conv }}$ will dominate over the radiation in the total energy flux $F=F_{\text {conv }}+F_{\text {rad }}$, thus allowing the latter to drop below the critical flux, $F_{\text {rad }} \lesssim F_{\text {crit }}$. Because the radiative force is less than gravity, such layers maintain an overall hydrostatic stratification with pressure and density decreasing outward. However, if such a super-Eddington condition applies near the surface - e.g., due either to an overall increase in luminosity, or to a local increase in opacity -, then convection may not be efficient enough to carry the required energy flux, and so the radiative flux may remain above critical. Because the radiative force would exceed gravity, this satisfies the local requirement for initiation of a steady-state outflow, or wind.

But simple energy arguments show that an outflow could not be globally sustained. An upper bound to the convective energy flux is set by

$$
F_{\text {conv }} \approx v_{\text {conv }} l d U / d r \lesssim a H d P / d r \approx a^{3} \rho,
$$

where $v_{\text {conv }}, l$, and $U$ are the convective velocity, mixing length, and internal energy density, and $a, H, P$, and $\rho$ are the sound speed, pressure scale height, pressure, and mass density. Setting this maximum convective flux equal to the total stellar energy flux $F=L / 4 \pi r^{2}$ yields an estimate for the maximum mass loss rate that could be initiated by radiative driving,

$$
\dot{M}_{\mathrm{max}, \mathrm{conv}} \equiv \frac{L}{a^{2}} \gg \dot{M}_{\mathrm{tiring}} \equiv \frac{L}{v_{\mathrm{esc}}^{2} / 2}=\frac{L}{\mathrm{G} M / R_{*}} .
$$

The latter inequality emphasizes that this exceeds by a large factor of $\sim v_{\text {esc }}^{2} / a^{2}$ the maximum mass loss that could be driven to escape the star's gravitational potential energy with all the available radiative energy, the socalled 'photon-tiring' limit (Owocki \& Gayley 1997). This implies that any such outflow initiated from the region that convection becomes inefficient would necessarily stagnate at some finite radius. One can imagine that the subsequent infall of material would likely form a complex spatial pattern, consisting of a mixture of both downdrafts and upflows, perhaps even resembling the 3-D cells of thermally driven convection. 
Overall, we thus see that a star that exceeds the Eddington limit is likely to develop a complex spatial structure, whether due to local instability to convection, or to global instability of flow stagnation. These instabilities are quite distinct from the thermodynamic instabilities of lower-mass stars, driven instead by the dynamical role of radiation momentum.

\subsection{Strange mode pulsation}

Analyses of the envelope structure for massive stars has over the years lead to the identification of a new kind of pulsational instability, now generally referred to as the 'strange-mode', which appears in complex modal analyses of stellar structure equations in domains that are quite distinct from those associated with thermodynamically driven pulsations of lower-mass stars. Subsequent analyses (e.g., Glatzel 1994; Glatzel et al. 1999) indicate that these are acoustic in nature, but with the radiation pressure playing a key role in both the oscillation and amplification. Indeed, it appears the excitation of these modes is most favored when the radiation pressure exceeds the gas pressure, which implies a region that is locally within a factor two of the Eddington limit. Whereas the thermodynamic instabilities of lower mass stars stem primarily from the trapping of energy in a local opacity increase, in strange modes opacity gradients play only an indirect role, for example in inducing evanescence in regions that bound the resonance cavity for the pulsation. The actual amplification of strange modes stems instead from work done on the gas by the radiation pressure when its perturbation lags in phase the perturbed density (Glatzel 1994). The strange mode can be manifest in both radial and non-radial pulsations (Glatzel \& Mehren 1996), and indeed there seems to be some good correspondence between domains of predicted strange-mode instability and observed photospheric line-profile-variability (Kiriakidis et al. 1993; Fullerton, Gies \& Bolton 1996). There are even suggestions that strange mode pulsations may play a role in driving or initiating stellar mass loss, e.g., for Wolf-Rayet stars (Kiriakidis et al. 1996) and/or Luminous Blue Variables (Glatzel 1997).

\subsection{Lateral instability of Thomson atmosphere}

Dating back to early work by Spiegel $(1976,1977)$ there have been speculations that atmospheres supported by radiation pressure would likely exhibit RayleighTaylor-type instabilities associated with support of a heavy fluid by a lighter one, leading to formation of 'photon bubbles'. Recent quantitative stability analyses by Spiegel \& Tao (1999) and by Shaviv (2001) do lead to the conclusion that even a simple case of a pure 'Thomson atmosphere' - i.e., supported by Thomson scattering of radiation by free electrons - would be subject to intrinsic instabilities for development of lateral inhomogeneities. The analysis by Shaviv (2001) suggests in particular that these instabilities share many similar properties to the excitation of strange mode pulsations. For example, they are favored when radiation pressure dominates over gas pressure, in this case operating in an intermediate regime between purely adiabatic and isothermal limits for the energy transport, wherein radiation diffuses against the opacity that shields localized gas compressions. With the dominance of radiation pressure, there is a tendency for it to compress the gas further, leading to an unstable growth of lateral structure. Shaviv (2001) identifies both stationary and propagating modes 
with maximum growth occurring at lateral scales comparable to the vertical scale height.

\subsection{Super-Eddington outflow modulated by porous opacity}

Shaviv $(1998,2000)$ has applied these notions of a laterally inhomogeneous radiatively supported atmosphere to suggest an innovative paradigm for how quasi-stationary wind outflows could be maintained from objects that formally exceed the Eddington limit. A key insight regards the fact that, in a spatially inhomogeneous atmosphere, the radiative transport will selectively avoid regions of enhanced density in favor of relatively low-density, 'porous' channels between them. This stands in contrast to the usual picture of simple 1-D gray-atmosphere models, wherein the requirement of radiative equilibrium ensures that the radiative flux must be maintained independent of the medium's optical thickness. In 2-D or 3-D porous media, even a gray opacity will lead to a flux avoidance of the most optically thick regions, much as in frequency-dependent radiative transfer in 1-D atmosphere, wherein the flux avoids spectral lines or bound-free edges that represent a localized spectral regions of non-gray enhancement in opacity.

A simple description of the effect is to consider a medium in which material has coagulated into discrete blobs of individual optical thickness $\tau_{\mathrm{b}}=\kappa \rho_{\mathrm{b}} l$, where $l$ is the blob scale, and the blob density is enhanced compared to the mean density of the medium by a volume filling factor $\rho_{\mathrm{b}} / \rho=(L / l)^{3}$, where $L$ is the interblob spacing. The effective overall opacity of this medium can then be approximated as

$$
\kappa_{\mathrm{eff}} \approx \kappa \frac{1-e^{-\tau_{\mathrm{b}}}}{\tau_{\mathrm{b}}} .
$$

Note that in the limit of optically thin blobs $\left(\tau_{\mathrm{b}} \ll 1\right)$ this reproduces the usual microscopic opacity $\left(\kappa_{\text {eff }} \simeq \kappa\right)$; but in the optically thick limit $\left(\tau_{\mathrm{b}} \gg 1\right)$, the effective opacity is reduced by a factor of $1 / \tau_{\mathbf{b}}$, thus yielding a medium with opacity characterized instead by the blob cross section divided by the blob mass $\left(\kappa_{\text {eff }}=\kappa / \tau_{\mathrm{b}}=l^{2} / m_{\mathrm{b}}\right)$. The critical mean density at which the blobs become optically thin is given by $\rho_{\mathrm{o}}=1 / \kappa h$, where $h=L^{3} / l^{2}$ is the characteristic length parameter for the porosity.

A key upshot of this is that the radiative acceleration in such a grey, but spatially porous medium would likewise be reduced by a factor that scales with the inverse of the mean density. In particular, for all $\rho(r)>\rho_{\mathrm{o}}$, the effective Eddington factor would be given by $\Gamma_{\text {eff }}=\Gamma \rho_{\mathrm{o}} / \rho$. Thus, for a formally super-Eddington atmosphere $\Gamma>1$, an effective transition from stratified medium to quasi-steady wind outflow could now occur where $\Gamma_{\text {eff }}=1$, yielding for the porous medium mass loss rate $\dot{M}=4 \pi R^{2} a \rho_{\mathrm{o}} \Gamma$, where $a$ is the sound speed at this location $r=R$. Simple algebraic manipulation shows that this porosity-mediated mass loss can be related to the tiring limit mass loss defined in eqn. (9) above,

$$
\dot{M}_{\text {por }}=\frac{a}{\mathrm{c}} \frac{R}{h} \dot{M}_{\mathrm{tiring}}
$$

Thus, as long as the porosity length is not too small, i.e., $h / R>a / \mathrm{c} \simeq 10^{-4}$, an outflow initiated in such a porous medium can be driven fully away from the star by the available radiative energy. 
While there remains much work to develop detailed 3-D models of spatial structure in such radiative atmospheres, Shaviv $(1998,2000,2001)$ has made an intriguing case for the relevance of porosity-mediated super-Eddington mass loss to both novae and LBVs. In this context, it is interesting to note that the estimated mass loss rate for $\eta$ Carinae during the 1840-1860 giant outburst seems very close to the photon-tiring-limited mass loss defined above.

\subsection{Rotational shaping of bipolar LBV nebula}

High-resolution pictures of the Homonuclus of $\eta$ Carinae show evidence of smallscale structure that might stem from instabilities in the original outburst. More prominently, this nebula exhibits a manifestly prolate form, with two lobes divided by a wispy equatorial 'skirt' that seems broken into radial spokes. From modelling of bipolar planetary nebula, a favored picture is that the two-lobe shape of $\eta$ Car results from spherical outburst that is confined at the equator by material in a preexisting circumstellar disk (Frank et al. 1998; Langer et al. 1999). A recently developed alternative scenario is that mass outburst itself was bipolar, perhaps because of rotational distortion of the source star (Owocki \& Gayley 1997; Maeder \& Meynet 2001). The gravity darkening of the rotational extended equatorial regions means that the radiatively driven mass loss tends to be stronger over the brighter stellar poles. The lower effective gravity of the equatorial regions also implies a slower wind outflow speed, leading naturally to an equatorial pinched form for the nebula (Dwarkadas \& Owocki 2002). Recent analysis of reflection spectra from the nebula seems indeed to favor the latter scenario, indicating that the current day wind from $\eta$ Car has a higher speed and higher mass flux over the pole (Smith et al. 2003).

Acknowledgments. This research was supported in part by NASA grant NAG5-3530 and NSF grant AST-0097983 to the Bartol Research Institute at the University of Delaware.

\section{References}

Abbott, D.C. 1980, ApJ 242, 1183

Babel, J., Montmerle, Th. 1997, A\&A 323, 121

Castor, J.I., Abbott, D.C., Klein, R.I. 1975, ApJ 195, 157 (CAK)

Carlberg, R.G. 1980, ApJ 241, 1131.

Cranmer, S.R. 1996, PhD thesis, University of Delaware, USA

Cranmer, S.R., Owocki, S.P. 1996, ApJ 462, 469

Dessart, L., Owocki, S.P. 2002, A\&A 383, 1113

Dwarkadas, V.V., Owocki S. 2002, ApJ 581, 1337

Feldmeier, A. 2001, Habilitation thesis, Universität Potsdam, BRD

Feldmeier, A., Puls, J., Pauldrach, A. 1997, A\&A 322, 878

Frank, A., Ryu, D., Davidson, K. 1998, ApJ 500, 291

Fullerton, A.W., Gies, D.R., Bolton, C.T. 1996, ApJS 103, 475

Gayley, K.G. 1995, ApJ 454, 410

Glatzel, W. 1994, MNRAS 271, 66

Glatzel, W. 1997, in: A. Nota \& H. Lamers (eds.), Luminous Blue Variables: Massive Stars in Transition, ASP-CS 120, 128 
Glatzel, W., Mehren, S. 1996, MNRAS 282, 1470

Glatzel, W., Kiriakidis, M., Chernigovskij, S., Fricke, K.J. 1999, MNRAS 303, 116

Joss, P.C., Salpeter, E.E., Ostriker, J.P. 1973, ApJ 181, 429

Kiriakidis, M., Fricke, K.J., Glatzel, W. 1993, MNRAS 264, 50

Kiriakidis, M., Glatzel, W., Fricke, K.J. 1996, MNRAS 281, 406

Langer, N., García-Segura, G., Mac Low, M.-M. 1999, ApJ (Letters) 520, L49

Lépine, S., Moffat, A.F.J., Henriksen, R. 1996, ApJ 466, 392

Lépine, S., Moffat, A.F.J. 1999, ApJ 514, 909

Lucy, L.B. 1984, ApJ 284, 351

Lucy, L.B., Solomon, P.M. 1970, ApJ 159, 879

MacGregor, K.B., Hartmann, L., Raymond, J.C. 1979, ApJ 231, 514

Maeder, A., Meynet, G. 2001, A\&A (Letters) 372, L9

Milne, E.A. 1926, MNRAS 86, 459

Oskinova, L.M., Ignace, R., Brown, J.C., Cassinelli, J.P. 2001, A\&A 372, 1009

Oskinova, L.M., Feldmeier, A., Hamann, W.-R. 2003, these Proceedings

Owocki, S.P. 1991, in: L. Crivellari, I. Hubeny, D.G. Hummer (eds)., Stellar Atmospheres: Beyond Classical Models (Kluwer: Dordrecht), p. 235

Owocki, S.P. 1999, in: B. Wolf, O. Stahl \& A.W. Fullerton (eds)., Variable and Nonspherical Stellar Winds in Luminous Hot Stars (Berlin: Springer), Lecture Notes in Physics 523, 294

Owocki, S.P., Castor, J.I., Rybicki, G.B. 1987, in: H. Lamers \& C.W.H. de Loore (eds.), Instabilities in Luminous Early-Type Stars (Dordrecht: Kluwer), p. 269

Owocki, S.P., Cranmer, S.R. 2002, in: C. Aerts, T.R. Bedding \& J. ChristensenDalsgaard (eds.), Radial and Non-radial Pulsations as Probes of Stellar Physics, ASP-CS 259, 512

Owocki, S.P., Gayley, K.G. 1997, in: A. Nota \& H. Lamers (eds.), Luminous Blue Variables: Massive Stars in Transition, ASP-CS 120, 121

Owocki, S.P., Puls, J. 1996, ApJ 462, 894

Owocki, S.P., Puls, J. 1999, ApJ 510, 355

Owocki, S.P., Rybicki, G.B. 1984, ApJ 284, 337

Owocki, S.P., Rybicki, G.B. 1985, ApJ 299, 265

Pittard, J. 2000, PhD thesis, University of Birmingham, UK

Runacres, M., Owocki, S.P. 2002, A\&A 381, 1015

Shaviv, N.J. 1998, ApJ (Letters) 494, L193

Shaviv, N.J. 2000, ApJ (Letters) 529, L137

Shaviv, N.J. 2001, ApJ 549, 1093

Smith, N., Davidson, K., Gull, T.R., Ishibashi, K. 2003, these Proceedings

Spiegel, E.A. 1976, in: R. Cayrel \& M. Steinberg (eds.), Physique des Mouvement dans les Atmospheres Stellaires (Paris: CNRS), p. 267

Spiegel, E.A. 1977, in: E.A. Spiegel \& J.-P. Zahn (eds.), Problems in Stellar Convection (Berlin: Springer), p. 19

Spiegel, E.A., Tao, L. 1999, Phys. Rep. 311, 163

ud-Doula, A., Owocki, S.P. 2002, ApJ 576, 413 


\section{Discussion}

HILLIER: In your rotating model with a magnetic field you had episodic ejection along the equatorial plane. Could this be used to explain the bullets and spokes seen in $\eta$ Carinae's ejecta?

OwockI: Perhaps. The simulations I showed were 2-D axis-symmetric, so these 'bullets' were formally 'rings'. But I expect in a full 3-D MHD model those rings would break up into 'bullets', or even 'spokes'. But the mass loss rate in $\eta$ Car is so high, that it would take a huge field $(\gg k G)$ to get this degree of magnetic confinement.

HuMPHREYS: Regarding $\eta$ Car, remember the Weigelt blobs within $0.22-0.3$ from the star. These compact knots were ejected in the 1890's lesser eruption and are in the equatorial plane. Your work addresses hot stars, but there is also considerable evidence for asymmetric, discrete mass loss ejections in the ejecta of very massive cool stars, probably for localized events.

Owocki: Yes, while most of mass loss in gravity-enhanced models is from the poles, there could be some equatorial wind compression. In particular, the inhibition of a wind compressed disk (WCD) by non-radial line forces would not apply to a continuum driven wind, which I believe the giant outburst must have been. As for cool stars, I will keep them in mind.

LANGER: By comparing our models for the $\eta$ Car Homunculus nebula, I am sure we can learn for both of them. I see one major morphological difference: we used the $\Omega$-limit in our model to obtain both the lobes and the 'skirt' of the Homunculus simultaneously. How do you think can you obtain the 'skirt' in the context of your model?

Owockr: Perhaps in conjunction with very rapid critical rotation, an equatorial Keplerian disk can also be centrifugally ejected, as seems to occur in, e.g., Be stars. Any outflow from such disk might then be the result of ablation by stellar radiation. Alternatively, for a continuum-driven wind, the WCD could still hold, since then the linedriven inhibition wouldn't apply. WCD models could indeed be favored in the gradual acceleration and/or slow outflow that might occur in a continuum-driven giant outburst.

GIES: Can you comment about the relative roles of magnetism and pulsation in Discrete Absorption Component (DAC) formation?

Owocki: I would distinguish between the classical DACs - which are extra absorption and probably stem from mass ejections - and Periodic Absorption Modulations (PAMs), which are modulations of the wind mass loss. I think the former are most likely magnetic in origin, and the latter tied to pulsations.

SCHAERER: Can you give us an update on the current status of wind instability models and their ability to predict X-ray luminosities in O-type stars?

Owocki: It seems that the observed soft X-ray flux can be accounted for by the clump-collision scenario, introduced by Achim Feldmeier, through the general scaling of $L_{\mathbf{x}} \propto L_{\mathrm{bol}}$, is not yet clearly explained. Regarding X-ray line profiles, those observed from $\zeta$ Pup seem in good agreement with what's expected from wind-shock emission. But the symmetric profiles observed from $\zeta$ Ori are a puzzle. 